\title{
Application of firefly algorithm for job shop scheduling
}

\author{
Guiying Mai ${ }^{1, a}$ \\ ${ }^{1}$ Gansu Normal University for Nationalities, Hezuo, Gansu, 747000, China \\ amaiguiying@163.com
}

Keywords: Firefly Algorithm; Job Shop Scheduling Problem; job splitting; Intelligent optimization algorithms

\begin{abstract}
An improved firefly algorithm is proposed for solving the job shop scheduling problem. Starting with the characteristic of job shop scheduling problem, combining disjunctive graph according to the sequence of scheduling, analyzing Scheduling options that decided by exchangeable set and alternative set, and then we can determine the optimal scheduling. Simulation results based on well-known benchmarks and comparisons with standard genetic algorithm demonstrate the effectiveness of the proposed bionic algorithm.
\end{abstract}

\section{Introduction}

It is indubitably that production scheduling plays a key role in the manufacturing systems of enterprises for maintaining a competitive position in fast-changing markets, so it is very important to develop effective and efficient advanced manufacturing and scheduling technologies and approaches to solve production scheduling. Job shop scheduling problems is a class of widely studied scheduling problems which has earned a reputation for being difficult to solve ${ }^{[1-5]}$, It is partly because job shop scheduling problems is non-polynomial (NP)-hard, and has proved to be a difficult task for planners and schedulers, particularly if optimal solutions are required.

More attention was paid on applying intelligent bionic algorithm to solve the problem in recently decades. Some scholars, which based on the simulation the behavior of swarm intelligence, presented for solving combinatorial optimization problems, Such as genetic algorithms, particle swarm optimization, ant colony algorithm, swarm algorithm and this paper will discuss the simulation of plant growth algorithm. Those intelligent algorithms have been widely used in many areas, also include job shop production scheduling problem ${ }^{[6-9]}$. In this paper, a new effective hybrid algorithm is proposed which is based on plant growth simulation algorithm for job shop scheduling with the criterion to minimize the maximum completion time.

\section{Job shop scheduling problem}

Job shop scheduling problem is the distribution of the task set which processing on the available machine in available time, in order to meet the prescribed performance targets set. Job shop scheduling problem is one of NP-hard problem. In fact, it is the process of operational decision making, which pre-optimizes the allocation of resources. The problem itself has diversity and complexity. Many scholars have a study and proposed a variety of solutions [8,9]. In our paper, Job shop scheduling mathematical model described as follows:

a) $J_{j}$ denotes the $j$ th job, $j=1,2, \ldots, n$;

b) $M_{i}$ denotes the $i$ th machine, $i=1,2, \ldots, m$;

c) $\mathrm{P}_{\mathrm{ij}}$ denotes the processing time of job $J_{j}$ on machine $M_{i}, j=1,2, \ldots, n ; i=1,2, \ldots, m$;

d) $\sigma^{j}$ denotes the sequence and technical constraints of the job $J_{j}$, for example the sequence of job $j$ which work on the machine $\sigma^{j}=\left(\sigma_{1}^{j}, \sigma^{j} 2, \ldots \sigma_{n}^{j},\right), \sigma^{j}{ }_{i} \in\{1,2, \ldots, m\}$ is the corresponding label of machine, $j=1,2, \ldots, n ; i=1,2, \ldots, m$;

e) $t_{i j}$ denotes the start time which the job $J_{j}$ works on the machine $M_{i}$;

f) $t_{j}$ denotes the completion time of the current operation $J_{j}$; 
Based on the above definition, we seek to minimize the production completion time as the objective function. Its mathematical model is $\min C_{\max }$.

\section{The basic principle of the firefly algorithm}

\subsection{The bionics principle of algorithm}

The firefly algorithm is the development of simulating biological characteristics of 1 the adults firefly, but the algorithm abandons some biological significance of luminous firefly, the firefly searches partners according to the search area only using the emission properties, and the firefly moves to position better firefly in the neighborhood structure, so as to realize the position evolution. In this algorithm, the firefly attracts one another reason depends on two factors, namely, its brightness and attraction. Among them, the firefly fluorescence brightness depends on the location of the target value, the brightness higher the location of the target value the better. The more light of fireflies has more attractive, it can attract brightness than its weak firefly in sight range to the direction of movement. If the fireflies have the same brightness, then they randomly move. The brightness and attraction are inversely proportional to the distance of the fireflies, they have increased as the distance decreases, which is equivalent to the simulation of the fluorescence characteristics of propagation in the space by the media absorption and fading. The firefly algorithm is adopted to simulate the firefly group behavior to construct a class of stochastic optimization algorithm. The bionic principle is: The points in the search space simulate the nature of firefly individuals, the search and optimization process simulation to attract and movement of the firefly individuals, the objective function of solving the problem is measured into the pros and cons of the location of the individual, the fittest of individual process for search and optimization process of a good analogy of feasible solutions to replace less feasible solutions.

\subsection{Mathematical description and analysis of the algorithm}

As mentioned above, the firefly algorithm includes two factors: the brightness and the degree of attraction. The brightness of the firefly reflects the pros and cons of location and determines its direction of movement, attraction degree determines the distance of the firefly mobile, constantly update the brightness and the attraction degree, so as to realize the goal of optimization. From a mathematical perspective describes the Firefly algorithm optimization mechanism, the description as follows ${ }^{[11,12]}$.

(1) The relative fluorescence intensity of fireflies as:

$$
h=h_{0} /\left(1+z r_{i j}^{2}\right)
$$

Among them: ${ }^{h}$ for the maximum fluorescence intensity of fireflies, that is itself $(r=0)$ fluorescence brightness, associated with the value of the objective function, the objective function value is better, its brightness is higher; $z$ as the light absorption coefficient, because the fluorescence decreases with the increasing of distance and media absorption ${ }^{[13]}$, so set the light absorption coefficient to reflect this characteristic, it can set to constant; $r_{i j}$ is the space distance of the firefly $i$ and the firefly $j$.

(2) The firefly attraction degree is:

$$
\rho=\rho_{0} * e^{-z r_{i j}^{2}}
$$

Among them: ${ }^{\rho_{0}}$ as the biggest attraction, namely the light source $(r=0)$ attraction degree; $z$, $r_{i j}$ meaning as above.

(3) The firefly $i$ is attracted to move to the firefly $j$, the location update by formula (3) decision.

$$
x_{i}^{\prime}=x_{i}+\rho^{*}\left(x_{j}-x_{i}\right)+\alpha^{*}(\text { rand }-1 / 2)
$$


Among them: $x_{i}^{\prime}$ is the individual $\mathrm{i}$ towards a brighter individual $\mathrm{j}$ update location, $x_{i}, x_{j}$ are the firefly $i$ and the firefly $j$ located before the entire population renewal; $\alpha$ is the step factor, it is constant between 0 and 1, rand is a random factor between 0 and 1,it obeys uniform distribution.

The process of optimization algorithm is: Firstly, the firefly populations randomly scattered in the solution space, each firefly has different fluorescence brightness at different positions, by comparing Eq.1, high brightness fireflies can attract low brightness fireflies to move, moving distance depending on the size of the attraction (according to Eq.2). In order to increase the search area, avoid getting into local optimization, and updated measures, according to the formula 3 to calculate the updated position. So by repeatedly movement, all individuals will be gathered in the highest brightness firefly position, so as to realize the optimization.

At present, it has been found that many insects existing Levy flight ${ }^{[14]}$, and Levy flight has been used in the field of optimization, and achieve the expected effect. In order to enhance the algorithm global search performance, avoid the population into a local optimum in the search process, in the firefly algorithm, if the individual is no better than their individual, choose to Levy flight instead of random flight in the original algorithm. In addition, the non - optimal those individuals in a population, the flight formula was improved: when they find more bright than their individual, first generates a random number $\mathrm{q}$ by the system, if $\mathrm{q}$ is less than 0.5 , the formula (4) is updated; otherwise, still use the formula (3) to update the individual position.

$$
x_{i}^{\prime \prime}=x_{i}^{\prime}+\rho^{*}\left(x_{j}-x_{i}^{\prime}\right)+\alpha^{*}(\text { rand }-1 / 2)
$$

Among them,: ${ }^{x_{j}}$ still expresses renewal position of individual $j$ before the entire population, ${ }_{i}^{\prime}$ expresses the individual $i$ toward the front of $j-1$ individuals than their bright individual after the update new position, $x_{i}^{\prime \prime}$ expresses $x_{i}^{\prime}$ toward than their bright individual $j$ after update location, $\rho$ expresses that individual $j$ appeal to the individual $i$. As can be seen, formula (4) is updated in real time, formula (3) depends only on the entire population before moving. This flight update can increase the randomness of flight, It is helpful to keep the diversity of population, increasing the population search space.

In order to accelerate the convergence of the population, the paper proposes a method of $\alpha$ updating, which $\alpha$ gradually decreases with increasing number of iterations. Update formula is as follows:

$$
\alpha=\alpha_{0}-e^{-0.001 * t}
$$

In the formula (5): $\alpha_{0}$ is $0.9, \mathrm{t}$ is number of iterations.

The concrete steps of the algorithm are as follows:

1) Initialization the basic parameter of algorithm. Set the number of fireflies is $s$, the biggest attraction is $\rho_{0}$, light absorption coefficient is $z$, the random parameters is $\alpha_{0}$, the maximum number of iterations is $t_{\max }=1000$, the evolving algebra $t=0$.

2) Randomly initialization firefly position, calculate the objective function value of firefly as the respective maximum fluorescence intensity of $h_{0}$.

3) By formula 1 and formula 2 to calculate the relative brightness of $h$ and attraction $\rho$, according to relative brightness of $h$ to decide the movement direction of the firefly.

4) The formula 5 is used to update $\alpha$, the individual, if there is more lighter than its individual, in accordance with the update is improved, by formula (4) to update the individual position; otherwise, the Levy flight is used to update the individual location.

5) According to the updated firefly position, to recalculate brightness of the firefly.

6) The system generates a random number, if the random number is less than the local search probability $p$, then local search of individuals of the population, and regenerate population.

7) Determine whether meet the conditions of termination of the algorithm. Such as the maximum number of iterations $t_{\max }$ is 1000 or best solution stagnation does not change, turn to step 7 , or $t=$ $t+1$, turn to step 3 . 
8) Output global extreme value point and optimal individual value.

\section{The experiment results and discussion}

To illustrate the effectiveness and performance of firefly algorithm for job shop scheduling to minimize makespan proposed in this paper, five different size representative Taillard instances taken from Internet based on practical data have been selected to compute. To examine the efficiency of the firefly algorithm, the comparisons are made with them and the standard GAs with different parameters. From the paper ${ }^{[10]}$ we can know that GAs with the LOX crossover, which mutation rate is 0.05 and initial population generated randomly, performed 20 runs on each problem instance for solving different size permutation flow shop scheduling, the result which we used Matlab language to program in this comparing computational experiment showed as follow:

Table 1 Comparison of statistical simulation results

\begin{tabular}{|c|c|c|c|c|c|c|}
\hline \multirow{2}{*}{$\mathrm{p}$} & \multirow{2}{*}{$\mathrm{n}, \mathrm{m}$} & \multirow{2}{*}{$\mathrm{C}^{* *}$} & \multicolumn{2}{|c|}{ Firefly algorithm } & \multicolumn{2}{c|}{ GA } \\
\cline { 3 - 6 } & & & $\mathrm{C}^{*}$ & $\%$ & $\mathrm{C}^{*}$ & $\%$ \\
\hline MT06 & 6,6 & 55 & 55 & 0 & 55 & 0 \\
\hline LA06 & 15,5 & 926 & 926 & 0 & 926 & 0 \\
\hline LA11 & 20,5 & 1222 & 1222 & 0 & 1222 & 0 \\
\hline MT10 & 10,10 & 930 & 945 & 4.45 & 980 & 10.22 \\
\hline MT20 & 20,5 & 1165 & 1182 & 4.80 & 1247 & 11.44 \\
\hline
\end{tabular}

Remark: in above table, the five problem are taken from Taillard ${ }^{[11]}$ which based on practical data; $N$ and $m$ denote the number of job and machine respectively; $\mathrm{C} * *$ indicate the optimal solution of the problem; $\mathrm{C} *$ is the optimal solution after 20 simulation computation; $\%$ denotes the average optimal value deviation relative to $\mathrm{C} * *$.

From simulation solution sees clearly that the optimal makespan of firefly algorithm is better than GAs and the stability of firefly algorithm are better than the GAs. Computational experiments show that the firefly algorithm for job shop scheduling to minimize makespan is very valid.

\section{Conclusions}

Although there are a number of literatures on firefly algorithm for engineer application field, the firefly algorithm for permutation flow shop scheduling does not have a literature. In this paper we have discussed a new approach based on firefly algorithm to solve permutation flow shop scheduling. Although it does not guarantee the optimality, such an approach provides solutions with good quality in a reasonable time limit, and compared with GAs. The performance of the new approach is evaluated in comparison with the results obtained from GAs for five representative instances. The obtained results show the effectiveness of the proposed approach.

\section{References}

[1] Li T,Wang C F ,et al.A global optimization bionics algorithm for solving integer Programming plant growth simulation algorithm[J ] .Systems Engineering Theory \& Practice, 2005,25(1):76 - 85.

[2] Li T,Wang Z t. Application of plant growth simulation algorithm on solving facility location problem [J ] .Systems Engineering Theory \& Practice, 2008, (12):107-115.

[3] Wang C ,Cheng H Z. Reactive power optimization base on plant growth simulation algorithm[J ]. Power System Technology,2006 ,30(21):37-41.

[4] Ding Xuefeng, Ma Liang, Ding Xuesong. The location allocation of logistics center of perishable products based on plant growth simulation algorithm[J]. Systems Engineering, 2009,27(2):96-101. 
[5] Pinedo M.Scheduling: theory, algorithms, and systems, 2rd Prentice-Hall, Englewood Cliffs, New Jersey. 2002, pp.126-144 [6] Marcelo Bertalmio, Luminita Vese. Image Filling-in in a Decomposition Space [J]. IEEE, 2003, (1):851 -853.

[6] Rao Y Q, Yan Z X, et al. Application of a Hybrid Genetic Algorithm for Job Shop Scheduling[J].Mechanical Science and Technology ,2006 , 25 (5) :584-587,607.

[7] Liu Y, Ye C M, Shen Y H. Solving the Job shop scheduling problem based on improved particle swarm algorithm [J]. Systems Engineering and Electronics, 2006 , 28 (4) :602-606.

[8] Colorni A, Dorigo M, Maniezzo V,et al. Ant system for job shop scheduling[J].Statistics and Computer Science, 1994, $341: 39-53$.

[9] Zhigang Lian, Bin Jiao,Xingsheng Gu. A similar particle swarm optimization algorithm for job shop scheduling to minimize makespan[J]. Applied mathematics and computation, 2006,183(2):1008-1017.

[10] Wang Li. Intelligent Optimization Algorithm and Application [M]. Beijing: Tsinghua University Press,2003.

[11] Taillard, E.: Benchmarks for basic scheduling problems. European Journal of Operational Research 1993(64), 278-285. 